\title{
Treatment in Elderly Glioblastoma Patients: General Reduction of Standard Therapy Should Be Avoided. Prognostic Value of MGMT Is Questionable*
}

\author{
Josef Pichler $^{1 \#}$, Isolde Hoellmüller ${ }^{1}$, Bahil Ghanim² ${ }^{2}$, Sabine Spiegl-Kreinecker ${ }^{1}$ \\ ${ }^{1}$ Department of Internal Medicine and Neurooncology, Landesnervenklinik, Linz, Austria; ${ }^{2}$ Medical University of Vienna, Vienna, \\ Austria. \\ Email: \#josef.pichler@gespag.at
}

Received July $16^{\text {th }}, 2013$; revised August $13^{\text {th }}, 2013$; accepted August $19^{\text {th }}, 2013$

Copyright (C) 2013 Josef Pichler et al. This is an open access article distributed under the Creative Commons Attribution License, which permits unrestricted use, distribution, and reproduction in any medium, provided the original work is properly cited.

\begin{abstract}
Purpose: Aim of this single center study was to determine whether elderly patients benefit from individualized treatment not excluding full standard therapy. Additionally predictive and prognostic factors influencing outcome in this patient's population were evaluated. Material and Methods: Between 1997 and 2010, 119 patients equal or older than 60 years were enrolled in this retrospective review. All patients had neuropathology confirmed diagnosis of glioblastoma. Treatment outcome concerning progression free survival was measured by MRI. For evaluation of O6-Methylguanin-DNA-methyltransferase (MGMT) Methylation-specific PCR was used. The log rank test and the Cox proportional hazards model were used to analyze the data. Factors considered in univariate and multivariate analyses included age, gender, Karnofsky performance scale (KPS), extent of resection, treatment with radio- and chemotherapy and MGMT status. Survival probabilities were estimated by means of the Kaplan Meier method. Results: Multivariate analysis demonstrated age, KPS and treatment more than surgery as prognostic factors for survival and sex, KPS, type of surgery and standard treatment as independent factors for PFS. MGMT status revealed no difference in survival between the methylated and unmethylated tumours in the whole study population (12.7 and 12.0, respectively). Surgery had an impact on survival with a significant advantage for complete resection. Conclusion: Extent of resection is essential even in elderly patients. Full standard treatment should be offered to elderly GBM patients with good clinical performance, there is no reason to withhold radio- or chemotherapy from these patients. MGMT promotor methylation of the tumour is not relevant for treatment decision.
\end{abstract}

Keywords: Glioblastoma; Elderly Patients; MGMT; Standard Therapy

\section{Introduction}

Glioblastoma (GBM) is the most frequent primary brain tumour with increased incidence in elderly people. Implementation of standard treatment with Radio/Chemotherapy for these tumours led to better outcome, but conclusive results for treatment decision in elderly patients could not be derived from the pivotal European Organisation of Research and Treatment of cancer (EORTC) trial [1]. Thus, years of discussions followed and resulted in several prospective trials in elderly brain tumour patients. The hypothesis behind all these trials was a diminished tolerance of standard treatment in this popula-

\footnotetext{
*Disclosure: The authors confirm that there are no disclosures, no conflicts of interest and no financial supports to declare.

\#Corresponding author.
}

tion. Geriatric Oncology is a relatively new field and addresses for the special issues and challenges in elderly patients. However, it is important to distinguish between chronologic and biologic age. Although differences in pharmacokinetics of cancer agents exist between older and younger patients, it is evident that healthy elderly patients benefit from and tolerate chemotherapeutic regimens like their younger counterparts [2]. Several articles of pharmacokinetics in elderly patients have shown no appreciable differences in pharmacokinetics of anticancer agents [3-5]. Functional status and co-morbidity are more sensitive predictors for outcome. Comprehensive geriatric assessment is useful but time consuming. Hence, reduction of treatment based on chronological age is questionable. Actually, cancer treatment develops 
further to personalized therapy looking on biomarkers. But, the above mentioned prospective studies generalize the patients only based on chronological age.

Our retrospective study reflects routine clinical work in elderly GBM patients where decisions are made by the treating physician according to the clinical condition rather than chronological age. Thus, the goal of our study was to determine whether elderly patients benefit from individualized treatment not excluding full standard therapy and prognostic and predictive factors influencing outcome in this patient's population.

\section{Patients and Methods}

One hundred and nineteen patients aged 60 years or older were enrolled in this retrospective study by querying our institutional neuro-oncological database. The patients were operated between 1997 and 2010 at the neurosurgery department of the Landesnervenklinik Linz, Austria, and had neuropathology confirmed diagnosis of glioblastoma. Informed consent was obtained from all patients. Treatment consisted of surgery, radiotherapy with concomitant and variable cycles of adjuvant chemotherapy with Temozolomide. As this review implemented patients over a long period of time standards of care changed and some patients had not received standard first line treatment.

Reasons for treatment termination were assessed. For overall survival (OS) analysis the cohort was divided into two groups, a younger group aged $60-68$ and the older group $\geq 69$ years. Survival status identified 111 deaths, six patients were alive, two patients were lost in followup and therefore 117 were eligible for evaluation. No other inclusion or exclusion criteria were applied. The extent of resection was defined by the surgeon.

Treatment outcome concerning progression free survival (PFS) was measured by magnetic resonance imaging (MRI) using our standard tumour protocol.

Data were collected on a 1.5 Tesla MR System (Siemens, Symphony) with the standard head coil. MRI included T2-transversal (T2), fluid attenuated inverse recovery (FLAIR) T1-transversal (T1), diffusion tensor imaging (DTI), Perfusion, T1-sagittal-Magnetization Prepared Rapid Gradient Echo (MPRAGE), T1-transversal with contrast agent (T1-KM).

For our purpose we evaluated the scans of all patients and calculated response and progression using McDonald criteria [6].

McDonald criteria suggest tumour size to be considered as the largest cross-sectional area. Response is calculated in four categories: complete response (CR): disappearance of all enhancing tumour on consecutive MRI scans at least 1 month apart, off steroids, and neurologically stable or improved. Partial response (PR): $\geq 50 \%$ reduction in size of enhancing tumour compared with the baseline scan on consecutive MRI scans at least 1 month apart, steroids stable or reduced, and neurologically stable or improved. Progressive disease (PD): $\geq 25 \%$ increase in size of enhancing tumour or any new tumour on MRI scans, or neurologically worse, and steroids stable or increased. Stable disease (SD): all other situations.

Evaluation of the $\mathrm{O}^{6}$-Methylguanin-DNA-mthyltransferase (MGMT) promotor methylation was done using DNA modification followed by Methylation-specific polymerase chain reaction (MSP). In brief, DNA was extracted from frozen GBM tumor tissue or paraffin embedded tissue using the QIAmp DNA Mini Kit and formalin fixated paraffin embedded (FFPE) Tissue Kit (Qiagen), respectively. DNA bisulfite conversion was performed with the Epitect Bisulfite Kit (Qiagen) according to the manufacturer's instruction. Methylation-specific PCR was done with primers specific for either methylated or unmethylated DNA as described previously [7].

\section{Statistical Analysis}

The log rank test and the more complex Cox proportional hazards model were used to analyse the data. Factors considered in the univariate and multivariate analyses included age, gender, KPS, extend of resection, treatment with radio- and chemotherapy and MGMT status. Overall survival and progression free survival were calculated according to the Kaplan Meier method.

\section{Results}

Patient's characteristics are listed in Table $\mathbf{1 .}$

Further details of therapy are summarized as follows with separate reporting of the two age groups (60-68/ 269): 77 patients $(42 / 35)$ received concomitant radiochemotherapy with Temozolomide, other first line chemotherapy, mostly CCNU, 18 (11/7), no radiotherapy 24 $(4 / 20)$. Reasons for treatment termination was assessed: progression of tumour in 21 patients $(16 / 5)$ seen in MRI scan, 5 patients with clinical progression (2/3), toxicity $18(6 / 12)$, one haematological toxicity resulted in death. Full therapy with 6 to 12 cycle's adjuvant Temozolomide was performed in 16 patients $(9 / 7)$. Other reasons for treatment termination in the remaining patients were 1) lost in follow up, 2) patients wish and 3) physician's decision due to clinical symptoms not related to progression. Second line therapy was given in 44 patients (31/ 13). The toxicity profile included the known side effects of radiotherapy and chemotherapy with comparable frequency and grade as published in the pivotal EORTC study [1].

The median overall survival (mOS) of the entire cohort was 12.0 months (CI 95\%: 10.8 - 13.3). However, a significant difference was observed between the younger 
Table 1. Patient and treatment characteristics.

\begin{tabular}{cc}
\hline Age & n (\%) $=\mathbf{1 1 9}$ \\
\hline mean & 69 years \\
$60-68$ & 57 \\
$\geq 69$ & 62 \\
Gender & \\
male & 77 \\
female & 42 \\
MGMT & \\
methylated & $70(59)$ \\
unmethylated & $49(41)$ \\
KPS & \\
mean & $(76)$ \\
$60-68$ & $(78)$ \\
$\geq 69$ & $(74)$ \\
Therapy & \\
Op & $29(24.4)$ \\
OP + RTX & $5(4.2)$ \\
OP + RTX + CTX & $85(71.4)$ \\
Surgery & \\
biopsy & $12(10)$ \\
PR & $59(50)$ \\
CR & $48(40)$ \\
\hline
\end{tabular}

Abbreviations: KPS, Karnofsky performance scale; MGMT, O6-Methylguanin-Methyltransferase; Op, operation; RTX, radiotherapy; CTX, chemotherapy; PR, partial resection; $\mathrm{CR}$, complete resection.

(60 - $68 \mathrm{y})$ and the older ( $\geq 69 \mathrm{y})$ age group $(\mathrm{p}=0.000$; mOS 17.6/9.1 months, respectively).

\subsection{MGMT Promoter Methylation Is Not Predictive for Improved Overall Survival}

MGMT status was collected from all GBM patients $(\mathrm{n}=$ $119)$ included in this study. Methylated MGMT sequences were detected in 70 of 119 (59\%) GBM tissues, whereas 49 GBM's (41\%) were completely unmethylated. Survival analysis using Kaplan-Meier curves showed no significant difference between patients harbouring MGMT methylated and unmethylated tumours $(\mathrm{p}=0.118$; median survival 12.7/12.0, respectively).

\subsection{Elderly Patients Profit from Complete Surgical Resection}

Surgery had an impact on survival with a significant advantage for complete resection (CR). No difference in outcome was shown between biopsy and partial resection (PR): CR vs biopsy, $\mathrm{p}=0.002$; biopsy vs $\mathrm{PR}, \mathrm{p}=0.213$; PR vs CR, $\mathrm{p}=0.036$.

\subsection{Clinical Performance Is Crucial for Outcome \\ Karnofsky performance status (KPS) was a significant}

factor for survival in univariate analysis (KPS $>80$, mOS: 15.1 months; KPS $<80$, mOS: 8.8 months; $\mathrm{p}=0.001$ ). Treatment had an essential impact on outcome; surgery alone: $\mathrm{mOS}$ 3.6; surgery and radiotherapy (RTX): mOS 20.1 months - these data should be interpreted with caution since there were only 5 patients in this group including unexpected long term survivors - surgery, radiotherapy and chemotherapy (CTX) mOS: 14.6 months.

\subsection{Progression Free Survival}

The type of surgery revealed a significant difference between biopsy/PR and CR (6.4/6.0 and 8.0, respectively, $p$ $=0.33-0.35)$. In contrast, a subgroup of patients with partial resection showed a difference in PFS. When only surgery was performed MGMT status had no prognostic impact on PFS, full treatment with RTX and CTX indicated a prognostic value (14.9 months in methylated and 12.0 in unmethylated tumours, $p=0.043$ ). See also Table 2. Kaplan Meier estimation (Figure 1) shows the relevant and significant outcome parameters for overall survival in terms of age, performance status, treatment and extent of resection. A significant difference in progression free survival related to extent of resection is also demonstrated. The curve indicating OS in relation to MGMT status shows no significance between MGMT methylated and unmethylated tumours but the rear section of the MGMT methylated curve displays a trend to a greater proportion of long term survivors in this group.

Table 2. Results for PFS and OS in univariate analysis (logrank test) estimated by Kaplan-Meier.

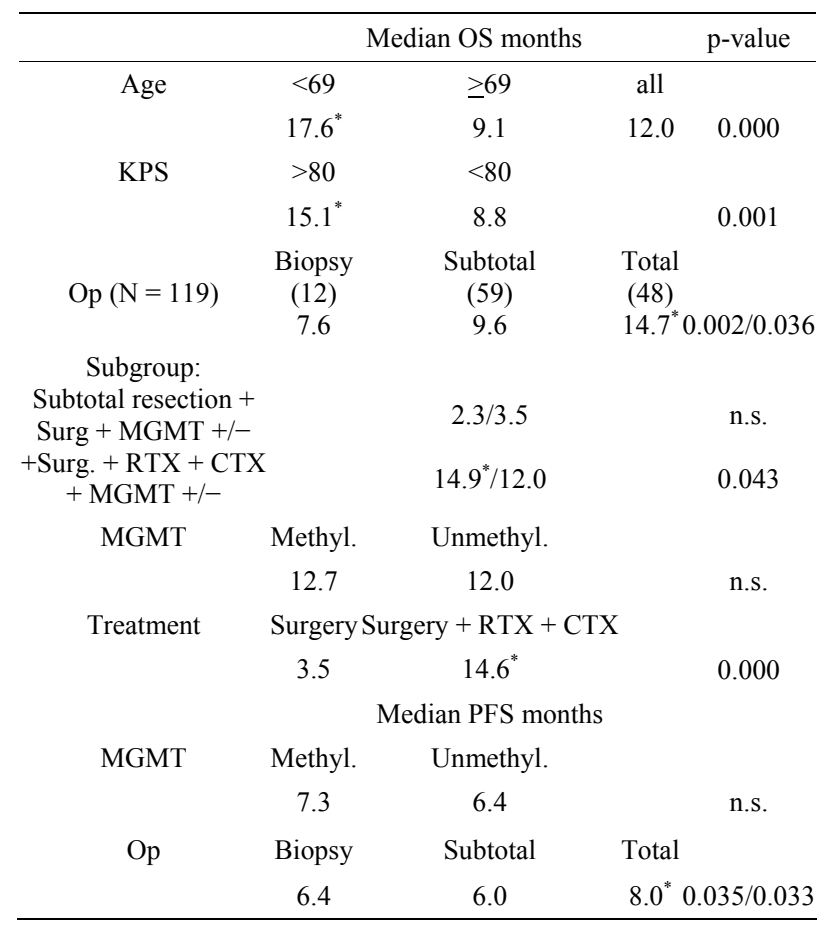



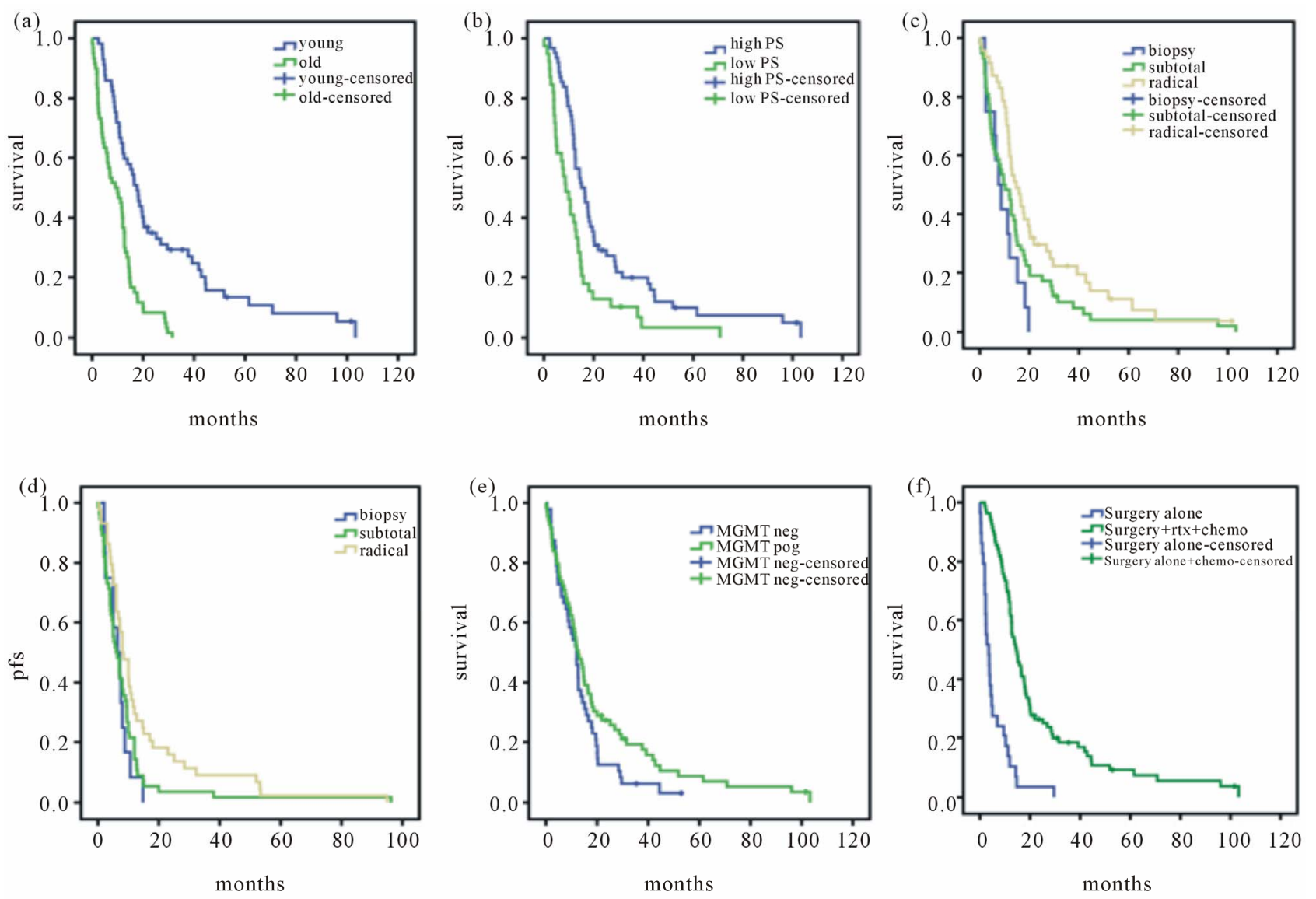

(a) OS by age (b) OS by KPS (c) OS by extent of rection

(d) PFS by extent of resection (e) OS by MGMT status (f) OS by treatment

Figure 1. Kaplan-Meier curves for different outcome parameters.

In multivariate Cox regression analysis age, KPS and treatment more than surgery showed independent prognostic quality for overall survival. With respect to PFS, sex, KPS, type of surgery and standard treatment were independent factors. The details are outlined in Tables 3 and 4. In terms of PFS MGMT status also showed no statistical difference (6.4 vs 7.2 in unmethylated or methylated tumours, respectively).

\section{Discussion}

The treatment of elderly GBM patients is still a matter of the subjective assessment of the authorized Neuro-oncologist and standard procedures are widely missing. Despite strong support of the literature for aggressive treatment in elderly patients [8] undertreatment in this patient cohort is the norm. Several studies confirmed the safety and efficacy of standard treatment in elderly patients [9]. Recently, three prospective studies were published considering the special issues in elderly patients. First, the Nordic glioma trial [10] compared 3 different treatment modalities including standard radiation consisting of 60 Gy, hypofractionated radiation with 34 Gy and chemotherapy with Temozolomide $200 \mathrm{mg} 5 / 28$ days every four weeks. Overall survival range in this study was 6.0 to 8.3 months. The NOA-08 trial [11] compared a dose dense chemotherapy with Temozolomide $100 \mathrm{mg} / \mathrm{m}^{2}$ one week on/one week off and standard radiotherapy with 60 Gy. OS was similar in both treatment groups with survival times of 8.6 and 9.6 months, respectively. Moreover, the ANOCEF study [12] treated patients with Temozlomide chemotherapy $150-200 \mathrm{mg} / \mathrm{m}^{2} 5 / 28$ days resulting in an OS of merely 25 weeks.

In comparison, mean age in the Nordic and NOA-08 trial was quite similar to our cohort (about 70 years), also clinical performance was comparable $(80 \%$ in NOA- 08 and $75 \%$ with WHO score $0-1$ in Nordic). The ANOCEF study included very old and frail patients and is not comparable with our cohort. In all of these studies the older GBM patients were treated using reduced treatment regimens and overall survival was shorter than in our patient group. When we look at the data from EORTC- 
Table 3. Cox regression overall survival. Multivariate analyses demonstrates significant results in terms of age, KPS, and treatment more than surgery (refer to the column: Significance), Hazard ratio (HR) is outlined separately.

\begin{tabular}{ccccccc}
\hline & B & SE & Wald & df & Significance & HR \\
\hline Sex & 0.076 & 0.237 & 0.102 & 1 & 0.750 & 1.079 \\
ageGroup & -0.811 & 0.268 & 9.194 & 1 & 0.002 & 0.444 \\
MGMTmeth & 0.236 & 0.228 & 1.074 & 1 & 0.300 & 1.266 \\
KPSgrouped & -0.870 & 0.238 & 13.407 & 1 & 0.000 & 0.419 \\
Op & & & & & \\
Total resection & & & 3.262 & 2 & 0.196 & 1 \\
Biopsy & 0.613 & 0.357 & 2.949 & 1 & 0.086 & 1.845 \\
Sutotal resection & 0.264 & 0.242 & 1.193 & 1 & 0.275 & 1.303 \\
Treatment & & & & & \\
Surgery alone & 1.634 & 0.325 & 25.243 & 1 & & 0.000 \\
Surg + RTX + CTX & & & 25.308 & 2 & 0.000 & 5.126 \\
\hline
\end{tabular}

Table 4. Cox regression PFS. Multivariate analyses demonstrates significant results in terms of sex, KPS, type of surgery and standard treatment (refer to the column: Significance), Hazard ratio (HR) is outlined separately.

\begin{tabular}{ccccccc}
\hline & B & SE & Wald & df & Significance & HR \\
\hline Sex & 0.488 & 0.236 & 4.277 & 1 & 0.039 & 1.630 \\
ageGroup & -0.165 & 0.234 & 0.499 & 1 & 0.480 & 0.847 \\
MGMTmeth & 0.355 & 0.233 & 2.066 & 1 & 0.151 & 1.398 \\
KPSgrouped & -0.500 & 0.224 & 4.954 & 1 & 0.026 & 0.607 \\
Op & & & & & \\
Total resection & & & 6.764 & 2 & 0.034 & 1.920 \\
Biopsy & 0.652 & 0.246 & 5.664 & 1 & 0.074 & 1.796 \\
Sutotal resection & 0.585 & 0.246 & 5.664 & 1 & 0.017 & \\
Treatment & & & & & & \\
Surgery alone & 1.489 & 0.305 & 23.775 & 1 & 0.000 & 0.000 \\
Surg + RTX + CTX & & & 24.071 & 2 & & 4.433 \\
\hline
\end{tabular}

NCIC trial [13] the subgroup analysis in this study revealed an OS of 10.9 to 11.8 months in the age group 60 to 70 with the two treatment arms radiotherapy alone or combined with chemotherapy. These results are equal to our data with a considerable difference of the population. In the EOR-TC-NCIC trial only patients were involved up to the age of 70 years.

Apart from the modified therapies used in these trials, type of surgery is also relevant and studies had shown that age alone has no impact on functional outcome [14, $15]$. In some centers possibly reluctance for extensive resection in elderly people is standard. For example about $40 \%$ in the NOA-08 trial had only biopsy, a well-known negative prognostic impact for outcome.

In our retrospective analysis the mean OS was 12.0 months, for the older group 9.6 and the younger 17.6. Elderly patients are a heterogenic population and probably no further study will be able to find the right treatment for everyone based on prospective evaluation. So, there's a need for a treatment algorithm, which can be addressed to the individual patient. Such an effort could be supported by retrospective data collected from NeuroOncology centers as cited above. Personalized medicine is coming up and should also be used in the elderly GBM patients, but not only based on molecular signatures but rather on clinical parameters. So it will be possible to avoid harmful therapy in frail people or to withhold potential effective therapy for fit patients.

Treatment with standard therapy is feasible also in elderly patients. We treated most of the patients with standard radiotherapy and concomitant chemotherapy and it was well tolerated.

Age was a prognostic factor, but also KPS and kind of treatment was relevant for outcome. In contrast to other known data of the EORTC trial [1] but in accordance with a study published recently [16], MGMT status was not prognostic in our study. An explanation could be, that we did not stratify the patients due to MGMT status upfront therapy. Nevertheless, an impact on survival became obvious as shown on the Kaplan Meier survival curve. The curves of OS in methylated vs unmethylated tumours indicated a separation of the lines in favor of a 
greater proportion of long term survivors, just a trend but maybe important. Additionally, no validated and standardized diagnostic tool for MGMT testing is available, multiple methods are used. Hence, in absence of an alternative first line therapy in MGMT negative GBM patients, MGMT remains largely a predictive factor, rather than a test on which therapies are based [17]. The high incidence of methylated tumours possibly relies on the fact that in our study MGMT status analysis was predominantly done using tumour specimens instead of formalin fixated paraffin embedded tissue used in multicenter studies. However elderly patients have probably different biological signatures. IDH-1 mutation is more common in young GBM patients [18] and telomerase activity and human telomerase reverse transcriptase have no prognostic impact in patients $>60$ years

Geriatric assessment for treatment decision is a useful tool, but we think, that KPS alone with keeping in mind relevant co-morbidities from anamnestic evaluation is sufficient enough.

Radiotherapy was well tolerated at full dose in the majority of our patients (75\%). Following surgery our patients were selected either for full therapy or best supportive care, in our experience the best clinical practice.

PFS as an important parameter for quality of life in GBM patients was higher in the group with complete tumor resection. Subtotal resection was also relevant for OS in the subgroup of patients with methylated MGMT promotor, only in this group MGMT had a prognostic and predictive impact.

\section{Conclusion}

Summing up our results, the extent of resection is essential even in elderly patients and is also important for the tolerance of subsequent radiotherapy. MGMT methylation of the tumor is probably not relevant for treatment decision considering the lack of alternative effective chemotherapies. Prospective trials are needed to evaluate the impact of the MGMT-status on the outcome for elderly patients treated according to the standard protocol with combined radio-/chemotherapy. But, the strong endpoint OS indicated that our intensive standard treatment modified by clinical parameters is superior to reduced treatment schedules. In conclusion, full standard treatment should be offered to elderly GBM patients with good clinical performance, since there is no reason to withhold radio- or chemotherapy from these patients. However, frail patients would deal best with supportive care as any further therapy will harm the patients without benefit for quality of life

\section{Acknowledgements}

The authors thank the collaborative Neuro-Oncology working group of the Landesnervenklinik Wagner Jauregg Linz for support.

\section{REFERENCES}

[1] R. Stupp, W. P. Mason, M. J. van den Bent, M. Weller, B. Fisher, M. J. Taphoorn, et al., "Radiotherapy Plus Concomitant and Adjuvant Temozolomide for Glioblastoma," New England Journal of Medicine, Vol. 352, 2005, pp. 987-996. doi:10.1056/NEJMoa043330

[2] T. L. Gillison and G. S. Chatta, "Cancer Chemotherapy in the Elderly Patient," Oncology (Williston Park), Vol. 24, No. 1, 2010, pp. 76-85.

[3] L. Balducci, "Pharmacology of Antineoplastic Medications in Older Cancer Patients," Oncology (Williston Park), Vol. 23, No. 1, 2009, pp. 78-85.

[4] A. Hurria and S. M. Lichtman, "Clinical Pharmacology of Cancer Therapies in Older Adults," British Journal of Cancer, Vol. 98, 2008, pp. 517-522. doi:10.1038/sj.bjc. 6604201

[5] S. M. Lichtman, "Pharmacokinetics and Pharmacodynamics in the Elderly," Clinical Advances in Hematology \& Oncology, Vol. 5, 2007, pp. 181-182.

[6] D. R. Macdonald, T. L. Cascino, S. C. Schold Jr. and J. G. Cairncross, "Response Criteria for Phase II Studies of Supratentorial Malignant Glioma," Journal of Clinical Oncology, Vol. 8, 1990, pp. 1277-1280.

[7] S. Spiegl-Kreinecker, C. Pirker, M. Filipits, D. Lotsch, J. Buchroithner, J. Pichler, et al., "O6-Methylguanine DNA Methyltransferase Protein Expression in Tumor Cells Predicts Outcome of Temozolomide Therapy in Glioblastoma Patients," Neuro-Oncology, Vol. 12, No. 1, 2010, pp. 28-36. doi:10.1093/neuonc/nop003

[8] J. G. Scott, J. H. Suh, P. Elson, G. H. Barnett, M. A. Vogelbaum, D. M. Peereboom, et al., "Aggressive Treatment Is Appropriate for Glioblastoma Multiforme Patients 70 Years Old or Older: A Retrospective Review of 206 Cases," Neuro-Oncology, Vol. 13, No. 4, 2011, pp. 428-436. doi:10.1093/neuonc/nor005

[9] S. E. Combs, J. Wagner, M. Bischof, T. Welzel, F. Wagner, J. Debus, et al., "Postoperative Treatment of Primary Glioblastoma Multiforme with Radiation and Concomitant Temozolomide in Elderly Patients," International Journal of Radiation Oncology, Biology, Physics, Vol. 70, 2008, pp. 987-992. doi:10.1016/j.ijrobp.2007.07.2368

[10] A. Malmstrom, B. H. Gronberg, C. Marosi, R. Stupp, D. Frappaz, H. Schultz, et al., "Temozolomide versus Standard 6-Week Radiotherapy versus Hypofractionated Radiotherapy in Patients Older than 60 Years with Glioblastoma: The Nordic Randomised, Phase 3 Trial," The Lancet Oncology, Vol. 13, No. 9, 2012, pp. 916-926. doi:10.1016/S1470-2045(12)70265-6

[11] W. Wick, M. Platten, C. Meisner, J. Felsberg, G. Tabatabai, M. Simon, et al., "Temozolomide Chemotherapy Alone versus Radiotherapy Alone for Malignant Astrocytoma in the Elderly: The NOA-08 Randomised, Phase 3 Trial," The Lancet Oncology, Vol. 13, No. 7, 2012, pp. 707-715. doi:10.1016/S1470-2045(12)70164-X 
[12] J. Gallego Perez-Larraya, F. Ducray, O. Chinot, I. CatryThomas, L. Taillandier, J. S. Guillamo, et al., "Temozolomide in Elderly Patients with Newly Diagnosed Glioblastoma and Poor Performance Status: An ANOCEF Phase II Trial," Journal of Clinical Oncology, Vol. 29, No. 22, 2011, 3050-3055.

[13] R. Stupp, M. E. Hegi, W. P. Mason, M. J. van den Bent, M. J. Taphoorn, R. C. Janzer, et al., "Effects of Radiotherapy with Concomitant and Adjuvant Temozolomide versus Radiotherapy Alone on Survival in Glioblastoma in a Randomised Phase III study: 5-Year Analysis of the EORTC-NCIC Trial," The Lancet Oncology, Vol. 10, No. 5, 2009, pp. 459-466. doi:10.1016/S1470-2045(09)70025-7

[14] K. L. Chaichana, K. K. Chaichana, A. Olivi, J. D. Weingart, R. Bennett, H. Brem, et al., "Surgical Outcomes for Older Patients with Glioblastoma Multiforme: Preoperative Factors Associated with Decreased Survival, Clinical Article," Journal of Neurosurgery, Vol. 114, No. 3, 2011, pp. 587-594. doi:10.3171/2010.8.JNS1081

[15] A. Mangiola, G. Maira, P. De Bonis, M. Porso, B. Pettorini, G. Sabatino, et al., "Glioblastoma Multiforme in the Elderly: A Therapeutic Challenge," Journal of NeuroOncology, Vol. 76, No. 2, 2006, pp. 159-163. doi:10.1007/s11060-005-4711-1

[16] K. Abhinav, K. Aquilina, H. Gbejuade, M. La, K. Hopkins and V. Iyer, "A Pilot Study of Glioblastoma Multiforme in Elderly Patients: Treatments, O-6-Methylguanine-DNA Methyltransferase (MGMT) Methylation Status and Survival," Clinical Neurology and Neurosurgery, Vol. 115, No. 8, 2013, pp. 1375-1378. doi:10.1016/i.clineuro.2012.12.023

[17] R. P. Thomas, L. Recht and S. Nagpal, "Advances in the Management of Glioblastoma: The Role of Temozolomide and MGMT Testing," Clinical Pharmacology: Advances and Applications, Vol. 5, No. 1, 2013, pp. 1-9.

[18] A. Leibetseder, M. Ackerl, B. Flechl, A. Wohrer, G. Widhalm, K. Dieckmann, et al., "Outcome and Molecular Characteristics of Adolescent and Young Adult Patients with Newly Diagnosed Primary Glioblastoma: A Study of the Society of Austrian Neurooncology (SANO)," NeuroOncology, Vol. 15, No. 1, 2013, pp. 112-121. doi:10.1093/neuonc/nos 283 